\title{
Reports
}

\section{MicroRNA Profiling in Benign and Malignant Conjunctival Melanocytic Lesions}

Conjunctival melanoma is a rare tumor with an incidence of 0.2 to 0.8 cases per 1 million people, although the incidence is increasing. Local recurrences and metastases are common. ${ }^{1}$ Up to $17 \%$ of the melanoma arise from nevi. ${ }^{2}$ In contrast to skin melanoma, for which many risk factors for a malignant course are known, the risk factors for conjunctival melanoma are currently not well understood, despite many attempts to predict the lesions' behavior by clinical and histopathologic characteristics and molecular parameters. ${ }^{1-3}$ A stringent follow-up and aggressive management is required to prevent significant morbidity and mortality, with only limited treatment options after a lesion has metastasized. ${ }^{2}$ These factors emphasize the need for informative biomarkers that can predict the lesion's behavior at an early stage of the disease.

During the last decades, microRNAs (miRNAs) have gathered interest with regard to their role in physiologic processes and can act either like a tumor suppressor or an oncogene. ${ }^{4}$ Because of the similarities between skin melanoma and conjunctival melanoma ${ }^{2}$ and the promising results of the involvement of miRNAs in skin melanoma, ${ }^{5}$ miRNAs may play an important role in the development of conjunctival melanoma as well.

In this study, we focused on discriminating miRNA levels in benign versus malignant conjunctival melanocytic lesions and differences in miRNA levels in the conjunctival melanoma with versus without metastases to determine whether it is possible to predict metastatic potential. For the first analysis, we used a discovery set consisting of 6 nevi and 20 melanoma cases, collected at the Department of Pathology, Erasmus MCUniversity Medical Center, Rotterdam, the Netherlands. For validation of the results, an independent validation cohort, consisting of 19 melanoma and 13 nevi cases from the University Hospital Leuven, Leuven, Belgium, was used. The nonmetastatic cases in the discovery set had a median follow-up of 7 years (range, 4.8-14.3 years for nevi and 0.1-21.4 years for conjunctival melanoma without metastases) and a median follow-up of 4 years (range, 0.6 to 6.9 years) for melanoma with metastasis. The patients from the University Hospital Leuven had a median follow-up for melanoma of 7 years (range, $0.0-15.0$ years) and for nevi of 0.3 years (range, 0.0-10.6 years); Table S1, available at www.aaojournal.org. Ethics committee approval was obtained. All research adhered to the tenets of the Declaration of Helsinki. Due to the noninterventional retrospective nature of the study informed consent was not required.

The relevant slides were reviewed and subsequently, tumorrich tissue was selected, containing at least $60 \%$ tumor cells, avoiding contamination with normal epithelium or inflammatory infiltrate. The formalin-fixed paraffin-embedded tissue, as is suitable for this analysis method, ${ }^{6}$ was cut, and the macrodissected tumor was dissolved in Trizol Reagent (ThermoFisher Scientific, PN 15596018; Bleiswijk, the Netherlands). Subsequently, total RNA was isolated using the Direct-zol RNA microPrep Kit from Zymo Research (Baseclear, art. no. R2062; Leiden, The Netherlands). After different quality checks, RNA samples of suitable quality (quantification cycle $[\mathrm{Cq}]$ value $<32$ for RNU48) were subjected to miRNA profiling using the TaqMan Low Density Array Card A (ThermoFisher Scientific) as described in the manufacturer's protocol.

The TaqMan miRNA array output data were uploaded in the ThermoFisher Cloud App and were analyzed using defined threshold settings for each individual miRNA. Because of the variability of the quality of the formalin-fixed paraffinembedded tissue samples, different normalization procedures were applied. After normalization, the data were analyzed using the statistical tools in QbasePlus (Zwijnaarde, Belgium) (MannWhitney $U$ test with correction for multiple testing). Amplification curves of potential targets were inspected in the ThermoFisher Cloud App and those exhibiting robust amplification were selected for further investigation using individual TaqMan assays. SPSS statistics software version 24 (SPSS, Inc; IBM-Netherlands, Amsterdam, The Netherlands) was used for constructing the receiver operating characteristic (ROC) curves and to calculate the area under the ROC curve.

We analyzed conjunctival nevi versus primary conjunctival melanoma with versus without metastasis. In the discovery cohort, miR-9-5p, miR-18b-5p, miR-196-5p, miR-425-5p, miR-450a-5p, miR-501-5p, and miR-615-3p showed significant differential levels, with a higher median Cq-value concerning the raw data for the melanoma group compared with the nevi group (Fig S1A [normalized data], Table S2 [raw data and normalized data], available at www.aaojournal.org). The miRNAs in the independent cohort as well as evaluating all samples combined revealed a similar pattern, with at least a $P$ value $\leq 0.001$ for miR-9-5p, miR196b-5p, miR-450a-5p, miR-501-5p, and miR-615-3p. Combining the 3 best performing miRNAs (miR-196b-5p, miR-615-3p, and miR-9-5p) resulted in an area under the ROC curve of 1.00 for the discovery cohort and of 0.98 for the independent validation cohort ( 0.983 and 0.972 for the combination of miR-615-3p and miR-9-5p, respectively; Table 1). No differences in miRNA levels were found in the melanoma group with versus without metastasis (Fig S1B, Table S2; available at www.aaojournal.org).

\section{Discussion}

In this study, we focused on upregulated miRNAs, because this finding is less influenced by factors like the amount and quality of the available tissue. This makes detecting upregulated miRNAs attractive for implementation in routine diagnostics. In 


\section{Ophthalmology Volume - Number -, Month 2019}

Table 1. MicroRNAs for Discrimination of Benign and Primary Malignant Melanocytic Lesions

\begin{tabular}{|c|c|c|c|c|}
\hline & \multicolumn{2}{|c|}{ Discovery Cohort } & \multicolumn{2}{|c|}{ Validation Cohort } \\
\hline & Nevi & Melanoma & Nevi & Melanoma \\
\hline & 6 & 20 & 13 & 19 \\
\hline & \multicolumn{2}{|c|}{ P Value/Fold Change } & \multicolumn{2}{|c|}{ P Value/Fold Change/Area under the ROC Curve } \\
\hline $\begin{array}{l}\text { MicroRNAs } \\
\text { miR-18b-5p } \\
\text { miR-196-5p } \\
\text { miR-425-5p } \\
\text { miR-450a-5p } \\
\text { miR-501-5p } \\
\text { miR-615-3p } \\
\text { miR-9-5p }\end{array}$ & & & & \\
\hline
\end{tabular}

\begin{tabular}{lll}
\hline & & Area under the ROC Curve \\
\hline Combined microRNAs & & \\
miR-196b-5p, miR-615-3p, and miR-9-5p & 1.000 & \\
miR-615-3p and miR-9-5p & 0.983 & 0.980
\end{tabular}

Individual TaqMan assays were carried out for samples from formalin-fixed paraffin-embedded tissues from the Erasmus University Medical Center (discovery cohort) and the University Hospital Leuven (validation cohort). Fold change for the melanoma and nevi cohorts and Mann-Whitney $U$ test results with correction for multiple testing $P$ values are from QbasePlus and the areas under the receiver operating characteristic (ROC) curve are from SPSS software version 24 .

daily practice, it can be very difficult to distinguish a benign from a malignant melanocytic lesion based on only histologic, immunohistochemical, and the well-known molecular findings. ${ }^{3}$ However, predicting the behavior of the melanocytic lesion is of high clinical importance. In this study, 5 miRNAs (of 377 miRNAs [1.3\%]) show increased levels in conjunctival melanoma compared with nevi, with combined higher levels of miR-9-5p, miR-196b-5p and miR-615-3p strongly associated with malignancy. This combination of miRNAs is certainly of interest with regard to the pathogenesis of conjunctival melanoma, in which a shared pathway for these miRNAs is suggested, possibly involving the homeobox gene clusters.? Furthermore, this combination may be of additional use in routine diagnostics to discriminate benign from malignant conjunctival melanocytic lesions, in case the amount of tissue or the currently available techniques seem to be insufficient. Additionally, miRNAs may be of interest with regard to targeted therapy. Unfortunately, we did not find miRNAs that could predict metastatic potential, emphasizing the need for further studies on this subject. MicroRNA profiling also will be of interest in the evaluation of primary acquired melanosis, because this condition is considered a risk factor for the development of melanoma ${ }^{1}$ and grading of primary acquired melanosis using a more objective method would have major consequences for both therapy and follow-up. However, to obtain reliable results, it is very important to avoid contamination of the atypical melanocytes with adjacent tissue. To overcome this problem, laser microdissection of single atypical melanocytes could be used in a research setting. However, such a laborious method would be less attractive for implementation in routine diagnostics. In the future, the diagnosis possibly may be made based on liquid biopsies in blood or tear film, rendering the histopathologic features no longer applicable.
Jolique A. van IPenburg, MD ${ }^{1}$

AD J.M. Gillis, ING ${ }^{2,3}$

LAMBert C.J. Dorssers, PhD ${ }^{2}$

Quincy C.C. VAN DEN Bosch, BSc ${ }^{1}$

Rita van Ginderdeuren, MD $^{4}$

Guy S. Missotten, MD, PhD

Nicole Naus, MD, PhD ${ }^{5}$

Dion PARIDAENS, MD, PhD ${ }^{5,6}$

Leendert H.J. Looijenga, PhD ${ }^{2,3}$,*

Robert M. VERDIJK, MD, PhD ${ }^{1,6,7, *}$

${ }^{1}$ Section Ophthalmic Pathology, Department of Pathology, Erasmus MC-University Medical Center, Rotterdam, the Netherlands;

${ }^{2}$ Department of Pathology, Laboratory for Experimental PathoOncology (LEPO), Erasmus MC-University Medical Center,

Rotterdam, the Netherlands; ${ }^{3}$ Princess Maxima Center for Pediatric Oncology, Utrecht, the Netherlands; ${ }^{4}$ Department of Ophthalmology and Pathology, University Hospital Leuven, Leuven, Belgium;

${ }^{5}$ Department of Ophthalmology, Erasmus MC-University Medical Center, Rotterdam, the Netherlands; ${ }^{6}$ The Rotterdam Eye Hospital, Rotterdam, the Netherlands; ${ }^{7}$ Department of Pathology, Leiden University Medical Center, Leiden, the Netherlands

*Both authors contributed equally.

Presented at: 30th European Congress of Pathology, September 2018, Bilbao, Spain.

Financial Disclosure(s): The author(s) have no proprietary or commercial interest in any materials discussed in this article.

Supported by De Stichting Wetenschappelijk Onderzoek Het Oogziekenhuis (grant no.: 2016-07). The funding organization had no role in the design or conduct of this research.

HUMAN SUBJECTS: Human subjects were included in this study. The human ethics committees Institutional Review Board approved the study. All research adhered to the tenets of the Declaration of Helsinki. 


\section{Reports}

Due to the noninterventional retrospective nature of the study no informed consent was required according to the Code of Conduct for Responsible Use of Human Tissue and Medical Research. Reference 67865 (Medical Ethics Committee, Erasmus MC-University Medical Center, Rotterdam, The Netherlands) and S60279 (European Data Protection Directive (95/46/EC, University Hospital Leuven, Leuven, Belgium).

No animal subjects were included in this study.

\section{Author Contributions:}

Conception and design: van Ipenburg, Gillis, Dorssers, van den Bosch, van Ginderdeuren, Missotten, Naus, Paridaens, Looijenga, Verdijk Analysis and interpretation: van Ipenburg, Gillis, Dorssers, van den Bosch, van Ginderdeuren, Missotten, Naus, Paridaens, Looijenga, Verdijk

Data collection: van Ipenburg, Gillis, Dorssers, van den Bosch, van Ginderdeuren, Missotten, Naus, Paridaens, Looijenga, Verdijk

Obtained funding: N/A

Overall responsibility: van Ipenburg, Gillis, Dorssers, van den Bosch, van Ginderdeuren, Missotten, Naus, Paridaens, Looijenga, Verdijk

Correspondence:

Robert M. Verdijk, MD, PhD, Department of Pathology, Erasmus MCUniversity Medical Center, Rotterdam, the Netherlands. PO Box 2040 3000CA, Rotterdam, the Netherlands. E-mail: r.verdijk@erasmusmc.nl.

\section{References}

1. Wong JR, Nanji AA, Galor A, Karp CL. Management of conjunctival malignant melanoma: a review and update. Expert Rev Ophthalmol. 2014;9(3):185-204.

2. Cao J, Heijkants RC, Jochemsen AG, et al. Targeting of the MAPK and AKT pathways in conjunctival melanoma shows potential synergy. Oncotarget. 2017;8(35):58021-58036.

3. Koopmans AE, Ober K, Dubbink HJ, et al. Prevalence and implications of TERT promoter mutation in uveal and conjunctival melanoma and in benign and premalignant conjunctival melanocytic lesions. Invest Ophthalmol Vis Sci. 2014;55(9):6024-6030.

4. Babapoor S, Wu R, Kozubek J, et al. Identification of miRNAs associated with invasive and aggressive phenotype in cutaneous melanoma by next-generation sequencing. Lab Invest. 2017;97(6):636-648.

5. Zhao G, Li Q, Wang A, Jiao J. YY1 regulates melanoma tumorigenesis through a miR-9 $\sim \mathrm{RYBP}$ axis. J Exp Clin Cancer Res. 2015;34:66.

6. Liu A, Xu X. MicroRNA isolation from formalin-fixed, paraffinembedded tissues. Methods Mol Biol. 2011;724:259-267.

7. Hoss AG, Kartha VK, Dong X, et al. MicroRNAs located in the Hox gene clusters are implicated in Huntington's disease pathogenesis. PLoS Genet. 2014;10(2):e1004188. 